\title{
Síntese de cerâmicas bifásicas de fosfato de cálcio pelo método Pechini
}

\author{
José Rosa de Souza Farias ${ }^{1 *}$ (1) \\ Geysivana Késsya Garcia Carvalho ${ }^{1}$ \\ Ycaro Breno Alves de Almeida ${ }^{1}$ \\ Josy Anteveli Osajima Furtini ${ }^{2}$ \\ Edson Cavalcanti Silva Filho ${ }^{2}$ \\ Aluska do Nascimento Simões Braga ${ }^{1,3}$
}

\section{Resumo}

Os fosfatos de cálcio $(\mathrm{CaP})$ são semelhantes com a parte inorgânica de uma matriz óssea, característica importante para um biomaterial. As fases de CaP mais amplamente utilizadas são a hidroxiapatita (HAp) e o beta fosfato tricálcico ( $\beta$-TCP). A associação da biocerâmica HAp/ $\beta$-TCP é uma alternativa promissora para uso na regeneração e enxertos ósseos. Assim, o objetivo deste trabalho foi sintetizar biocerâmicas de HAp/ $\beta$-TCP via método Pechini, avaliando a influência de diferentes solventes na síntese. As caracterizações foram realizadas por difração de raios X (DRX), espectroscopia de infravermelho com transformada de Fourier (FTIR), análises termogravimétricas (TG/DTG) e microscopia eletrônica de varredura (MEV). Os resultados comprovaram que o método de síntese é eficiente na formação da cerâmica bifásica de HAp/ $\beta$-TCP, independentemente do tipo de solvente utilizado, água ou álcool etílico, resultando na formação de nanopartículas agrupadas de aspecto morfológico poroso e formado por grãos irregulares.

Palavras-chave: Hidroxiapatita; Beta fosfato tricálcico; Pechini.

\section{Synthesis of calcium phosphate biphasic ceramics by Pechini method}

\begin{abstract}
Calcium phosphates $(\mathrm{CaP})$ are similar to the inorganic part of a bone matrix, an important characteristic for a biomaterial. The most widely used $\mathrm{CaP}$ phases are hydroxyapatite (HAp) and tricalcium beta phosphate ( $\beta$-TCP). The combination of biochemistry HAp $/ \beta-\mathrm{TCP}$ is a promising alternative for use in bone regeneration and grafting. Thus, the objective of this work was to synthesize HAp/ $\beta$-TCP bioceramics via Pechini method, evaluating the influence of different solvents on synthesis. The characterizations were performed by X-ray diffraction (XRD), Fourier transform infrared spectroscopy, thermogravimetric analysis (TG/DTG) and scanning electron microscopy (SEM). The results proved that the synthesis method is efficient in the formation of biphasic ceramic of HAp/ $\beta$-TCP, regardless of the type of solvent used, water or ethyl alcohol, resulting in the formation of grouped nanoparticles with a porous morphological aspect and forming irregular grains.
\end{abstract}

Keywords: Hydroxyapatite; Tricalcium beta phosphate; Pechini.

\section{Introdução}

As biocerâmicas de fosfato de cálcio $(\mathrm{CaP})$ vêm sendo extensivamente estudadas nas últimas décadas, em virtude da sua importância no tratamento e reparo de partes danificadas do tecido ósseo. Porém o tratamento clínico ainda é um grande desafio [1-3], uma vez que a utilização desse material deve cumprir requisitos biológicos e permitir a revitalização no sítio de implantação [4].

Com base na composição, os principais fosfatos de cálcio sintéticos usados atualmente como biomateriais são classificados como hidroxiapatita (HAp), fosfato beta-

${ }^{1}$ Departamento de Engenharia de Materiais, Universidade Federal do Piaui - UFPI, Teresina, PI, Brasil.

${ }^{2}$ Departamento de Quimica, Universidade Federal do Piaui - UFPI, Teresina, PI, Brasil.

${ }^{3}$ Programa de Pós-graduação em Engenharia de Materiais, Instituto Federal do Piauí-IFPI, Teresina, PI, Brasil.

*Autor correspondente: josefarias2100@gmail.com

2176-1523 (C) 2021. Farias et al. Publicado pela ABM. Este é um artigo publicado em acesso aberto (Open Access) sob a licença Creative Commons Attribution, que permite uso, distribuição e reprodução em qualquer meio, sem restrições desde que o trabalho original seja Commons Attribution,
corretamente citado. 
tricálcico ( $\beta$-TCP) e fosfatos de cálcio bifásicos (BCPs) para misturas de HAp e $\beta$-TCP [5]. Os BCPs podem ser aplicados para administração de medicamentos, substitutos do tecido ósseo e em aplicações dentárias, devido à sua biocompatibilidade e biodegradabilidade, bem como por sua eficácia na regeneração de tecidos duros em oposição a HAp pura ou $\beta$-TCP puro [6]. A HAp é uma fase mais estável, enquanto $\beta$-TCP é mais solúvel, os biomateriais de BCPs são frequentemente preferidos, pois permitem adaptar um equilíbrio adequado entre reabsorção e solubilização [7].

Embora as biocerâmicas $\mathrm{CaP}$ tenham muitas vantagens, elas também sofrem desvantagens como, baixa resistência mecânica, presença de impurezas, tamanho de grão em escala micro e tamanho e forma de partículas não homogêneas. No entanto, nos últimos anos, várias modificações nos parâmetros de fabricação, como temperatura sinterização, tempo de imersão, $\mathrm{pH}$ e pureza dos materiais iniciais deram origem a biomateriais com propriedades físico-químicas aprimoradas [8]. Consequentemente, vários pesquisadores se concentraram na síntese CaP. Até o momento, vários métodos de síntese são utilizados para a produção, incluindo sol-gel [8-11], processo hidrotérmico [12,13], técnicas de precipitação [14,15], reação em estado sólido [16,17] e deposição $[18,19]$ entre outros. O método Pechini é uma síntese desenvolvida do aprimoramento do método sol-gel, onde o processo se baseia na formação de citratos metálicos em solução, ocorrendo entre íons metálicos e íons de hidroxilas e carboxilas. Quando aquecidos após a adição de um diol, os citratos são reticulados para criar uma resina polimérica rígida por poliesterificação, na qual os cátions são distribuídos uniformemente no nível atômico [20]. A resina é queimada a uma temperatura moderada para pirolisar e se livrar das substâncias orgânicas. Finalmente, pode ser obtida uma mistura fina de óxidos metálicos. A vantagem notável do processo Pechini é que ele pode ser usado para preparar materiais de composição complexos homogêneos [21-23].

Apesar de tais atrativos, tal método é pouco discutido na literatura para a síntese de cerâmicas de $\mathrm{CaP}$, com isso, é produzido uma série de lacunas quanto a influência das variáveis e parâmetros de processo em sua produção, dentre as quais se pode destacar: o uso de diferentes solventes. Dessa forma, este trabalho teve por objetivo sintetizar fosfatos de cálcio compostos por HAp e $\beta$-TCP pelo método Pechini, avaliando os solventes água e álcool etílico, e como estes podem influenciar nas características finais do material sintetizado.

\section{Materiais e métodos}

\subsection{Materiais}

Foram utilizados os reagentes: ácido cítrico anidro $\left(\mathrm{C}_{6} \mathrm{H}_{8} \mathrm{O}_{7}\right)$ e nitrato de cálcio $\left(\mathrm{Ca}\left(\mathrm{NO}_{3}\right)_{2} \cdot 4 \mathrm{H}_{2} \mathrm{O}\right)$ fornecidos pela Cinética; fosfato de amônio bibásico, $\left(\left(\mathrm{NH}_{4}\right)_{2} \mathrm{HPO}_{4}\right)$ da Neon; etileno glicol $\left(\mathrm{HOCH}_{2} \mathrm{CH}_{2} \mathrm{OH}\right)$, hidróxido de sódio
$(\mathrm{NaOH})$, álcool etílico absoluto $\left(\mathrm{CH}_{3} \mathrm{CH}_{2} \mathrm{OH}\right)$ produzidos pela Synth e água deionizada.

\subsection{Síntese da biocerâmica de HAp/ß-TCP (BCP)}

Inicialmente, foi realizado a dissolução de ácido cítrico (AC) em $100 \mathrm{ml}$ do solvente estudado: água neutro, água basificada com pH 10 (corrigido com $\mathrm{NaOH}$ ) ou álcool etílico. A solução foi mantida sob agitação constante com temperatura máxima de $70^{\circ} \mathrm{C}$. Os cátions metálicos (CM), nitrato de cálcio e fosfato de amônio bibásico foram acrescentados nas proporções AC/CM de 3:1. Para iniciar às reações de esterificação e poliesterificação, adicionou-se o etileno glicol (EG) na proporção 60/40 para AC/EG, em seguida a temperatura foi elevada a $120^{\circ} \mathrm{C}$, formando o gel.

A resina formada passou por tratamento térmico (pirólise) em forno mufla na temperatura de $400^{\circ} \mathrm{C} / 1 \mathrm{~h}$, com uma taxa de aquecimento de $10^{\circ} \mathrm{C} / \mathrm{min}$. O puff formado foi desaglomerado e passado em peneira ABNT n ${ }^{\circ} 200(0,074 \mathrm{~mm})$. $\mathrm{O}$ pó resultante foi calcinado na temperatura $1000^{\circ} \mathrm{C}$, com taxa de aquecimento de $10^{\circ} \mathrm{C} / \mathrm{min}$ durante $1 \mathrm{~h}$, para a formação da cerâmica bifásica. As amostras sintetizadas foram denominadas de CaP-base, CaP-neutro e CaP-etil, quando do uso da água com $\mathrm{pH}$ básico, água com $\mathrm{pH}$ neutro e álcool etílico como solventes, respectivamente.

\subsection{Caracterizações}

A difração de raios X (DRX) realizada nas amostras foram executados no equipamento da Shimadzu, modelo XRD-6000, utilizando radiação $\mathrm{Cu}-\mathrm{K} \alpha(40 \mathrm{kV} / 30 \mathrm{~mA})$, varredura de $20^{\circ}$ a $60^{\circ}$, com velocidade de $2^{\circ} /$ min em intervalos de $0,02^{\circ}$ e tempo de 0,6 segundos. Para identificação e quantificação das fases utilizou-se o software X'pert Panalytical High Score Plus e o banco de dados JCPDF (Joint Committee on Powder Diffraction and Standards). A cristalinidade foi obtida no programa da Shimadzu Cristalinity, o qual se utilizou o coeficiente de correção de Lorentz. O cálculo do tamanho de cristalito foi realizado a partir do pico da reflexão basal de maior intensidade por meio da deconvolução do pico por meio da Equação 1 conhecida como equação de Scherrer [22].

$$
D=\frac{K \cdot \lambda}{\beta \cdot \operatorname{Cos} \theta}
$$

Na formulação, D corresponde ao tamanho médio dos cristalitos, $\mathrm{K}$ é a constante da forma dos cristais, que para o caso analisado corresponde a 0,90 . $O$ valor de $\beta$ equivale à largura meia altura do pico de difração e por fim $\theta$ é o ângulo de Bragg do pico difratado.

A espectroscopia de infravermelho com transformada de Fourier (FTIR) foi realizada em um espectrômetro BunKer modelo TENSOR 27, com intervalo de 400 a $4.000 \mathrm{~cm}^{-1}$, para cada amostra preparada com pastilha de $\mathrm{KBr}$. 
AAnálise termogravimétrica (TG/DTG), foi realizada em um analisador térmico, modelo TA-60, da Shimadzu, com taxa de aquecimento de $10^{\circ} \mathrm{C} / \mathrm{min}$, em atmosfera de nitrogênio, em faixa de temperatura do ambiente $\left(25^{\circ} \mathrm{C}\right)$ até a temperatura máxima de $1000^{\circ} \mathrm{C}$.

A microscopia eletrônica de varredura (MEV), foi realizada em um microscópio modelo Auriga da Carl Zeiss.

\section{Resultados e discussão}

A Figura 1 exibe os difratogramas de raios $\mathrm{X}$ das amostras CaP-base, CaP-neutro e CaP-etil, respectivamente.

Observando os difratogramas da Figura 1, percebe-se que independente do solvente utilizado o padrão é semelhante para todas as amostras, com formação de duas fases, sendo estas: a hidroxiapatita (JPDS 09-0432) e o beta fosfato tricálcico (JPDS 09-0169). A CaP-base apresentou a maior cristalinidade (Tabela 1) e um aumento na quantidade de hidroxiapatita formada em relação as amostras CaP-neutro e CaP-etil. Isso ocorreu, possivelmente, pois no $\mathrm{pH}$ alcalino o grupo funcional hidroxila desprende-se do $\mathrm{Na}^{+}$, unindo-se ao $\mathrm{Ca}^{2+}$ e ao $\mathrm{PO}_{4}^{3-}$, favorecendo a razão molar para produção de hidroxiapatita $[24,25]$

A amostra CaP-etil apresentou uma redução na intensidade dos picos localizados $28,9^{\circ}(120) ; 31,75^{\circ}$ (121); $32,15^{\circ}$ (112); $34,02^{\circ}(002)$ e $48,60^{\circ}(230)$, que estão relacionados a fase HAp. Provavelmente, isso ocorreu, pois o álcool etílico possui um ponto de ebulição $\left(78,3^{\circ} \mathrm{C}\right)$ menor do que o da água $\left(100^{\circ} \mathrm{C}\right)$, acelerando assim a reação, o que proporcionou um rápido aumento na viscosidade da solução, dificultando a difusão dos íons [26]. A maior quantidade de fase $\beta$-TCP, quando do uso do álcool etílico como solvente, pode estar ainda associada a uma hidroxiapatita não estequiométrica (uma variação da proporção $\mathrm{Ca} / \mathrm{P}=1,67$ ), originária da má complexação do grupo $\mathrm{PO}_{4}^{3-}$, resultante da homogeneização insuficiente na distribuição de íons na solução [27].

A literatura reporta que o uso de cerâmicas bifásicas de HAp/ $\beta$-TCP tem um enorme impacto na capacidade de indução óssea in vivo devido à sua solubilidade diversificada no ambiente fisiológico e no microambiente iônico local, alcançando a formação ideal do crescimento do tecido ósseo, além disso resistência e tenacidade à fratura podem ser melhoradas com uma quantidade baixa da fase $\beta-\mathrm{TCP}[28,29]$.

A Tabela 1 exibe os resultados para tamanho médio de cristalito, cristalinidade e percentual de fases das amostras CaP-base, CaP-neutro e CaP-etil.
Todas as amostras apresentaram elevados valores de cristalinidade, variando de 88,2 à $96 \%$, sendo estes superiores aos valores relatados na literatura para síntese dessas biocerâmicas [27], o que pode tornar o material promissor na aderência do tecido e crescimento dos tecidos sobre os implantes que sejam revestidos com essas biocerâmicas. Percebe-se que as amostras que utilizaram água com $\mathrm{pH}$ neutro ou água com $\mathrm{pH}$ básico como solvente foram mais

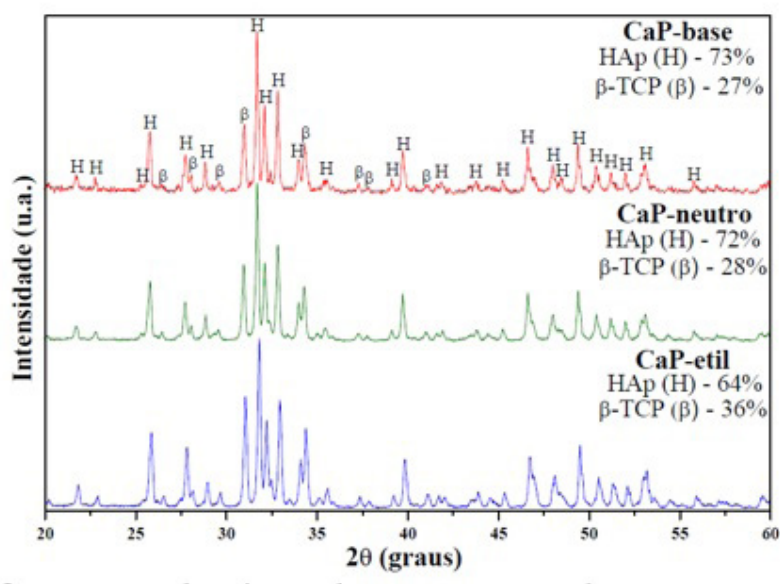

Figura 1. Difratogramas de raios $\mathrm{X}$ das amostras $\mathrm{CaP}-$ base, $\mathrm{CaP}-$ neutro e CaP-etil.

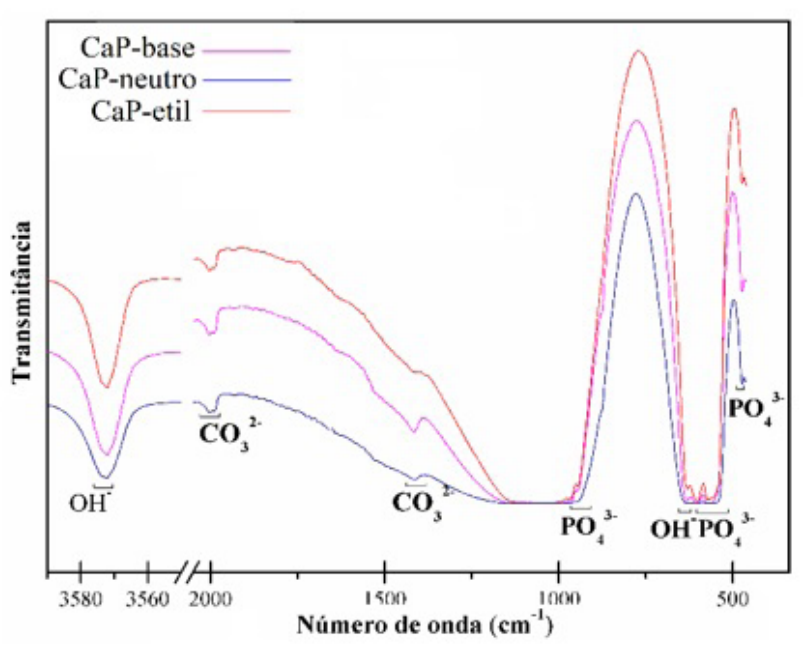

Figura 2. Espectros de FTIR para CaP-base, CaP-neutro e CaP-etil.

Tabela 1. Percentual de fases, tamanho de cristalito e cristalinidade das amostras CaP-etil, CaP-água e CaP-base

\begin{tabular}{ccccccc}
\hline \multirow{2}{*}{ Amostras } & \multicolumn{3}{c}{ Fases (\%) } & & Tamanho do & \multirow{2}{*}{ Cristalinidade (\%) } \\
\cline { 2 - 4 } & HAp & $\boldsymbol{\beta}$-TCP & & Cristalito (nm) & 96 \\
CaP-base & 73 & 27 & 53,6 & 93,4 \\
CaP-neutro & 72 & 36 & 43,9 & 88,2 \\
CaP-etil & 64 & & 45,8 & \\
\hline
\end{tabular}


cristalinas, pois a evaporação lenta do solvente dificulta o processo de segregação do grupo de $\mathrm{PO}_{4}^{3}$, favorecendo a produção e a cristalização da hidroxiapatita [24].

Os valores médios do tamanho de cristalito variaram de 43,09 a 53,6 nm. Esses valores de tamanho de cristalito estão compatíveis com os valores encontrados na literatura para síntese de hidroxiapatita através de outras rotas sol-gel $[30,31]$.

A Figura 2 exibe os espectros do FTIR das amostras $\mathrm{CaP}$-base, $\mathrm{CaP}$-neutro e CaP-etil, respectivamente. Para todas as amostras percebe-se a existência de três grupos principais de composição: $\mathrm{OH}^{-}, \mathrm{PO}_{4}{ }^{3-}$ e $\mathrm{CO}_{3}{ }^{2-}$. As bandas que provam a presença da HAp são 635 e $3.572 \mathrm{~cm}^{-1}$, correspondente ao alongamento da vibração do grupo da hidroxila $\left(\mathrm{OH}^{-}\right)$, indicando a existência de moléculas de água fisicamente absorvidas [32]. No entanto, essa banda não pode ser vista na fase de $\beta$-TCP, pois sua estrutura química não possui o grupo $\mathrm{OH}^{-}$. A banda característica para o grupo do fosfato $\left(\mathrm{PO}_{4}^{3-}\right)$ da fase $\beta$-TCP aparece em $947 \mathrm{e} 973 \mathrm{~cm}^{-1}\left(v_{1}\right)$ [8]. As bandas do grupo $\mathrm{PO}_{4}^{3-}$ da fase HAp ocorrem em $603 / 475 \mathrm{~cm}^{-1}\left(v_{4}\right)$, $570 \mathrm{~cm}^{-1}\left(v_{2}\right)$ e $961 \mathrm{~cm}^{-1}\left(v^{1}\right)$ [21,33]. As bandas $1.414 \mathrm{e}$ $1.993 \mathrm{~cm}^{-1}$ (v3) pertencem ao grupo dos carbonatos $\left(\mathrm{CO}_{3}{ }^{2-}\right)$ e são atribuídas a posições de fosfato substituída por $\mathrm{CO}_{3}^{2-}$ da rede de HAp [34]. A banda em $1.637 \mathrm{~cm}^{-1}$ corresponde à água absorvida, ocorrendo devido à vibração de alongamento da água livre [35].

Observa-se que para a CaP-base ocorre uma redução nas bandas 947 e $973 \mathrm{~cm}^{-1}$, confirmando a redução da fase $\beta$-TCP quando do uso da água $\mathrm{pH}$ básico como solvente, corroborando com os resultados do DRX.

Analisando as micrografias percebe-se que para todas as amostras não é possível fazer distinção entre as fases de HAp e $\beta$-TCP. Nota-se que as partículas estão agrupadas formando agregados de formato irregular, independente do solvente usado. Todos os pós produzidos são altamente reativos, sendo isto comprovado pela formação desses agregados. Porém, a amostra CaP-etil apresenta, aparentemente, um menor grau de aglomeração das partículas. Pei et al. [36] também perceberam essa mesma diferença no grau de aglomeração das partículas ao estudarem a água e o álcool etílico como solventes na síntese de $\mathrm{NiO}$.
A morfologia (Figura 3) obtida é porosa, em todas as amostras, isto é característico do método de síntese que utiliza muita matéria orgânica, resultando em tal porosidade quando esta é eliminada na etapa de sinterização. A formação de poros é benéfica, pois permite a circulação de fluido fisiológico quando utilizado como biomaterial [37].

A Figura 4 exibe as curvas de TG/DTG das amostras, sintetizadas usando álcool etílico (a), água pH neutro (b) e água pH básico (c) como solventes, respectivamente.

Analisando as curvas da Figura 4 percebe-se uma similaridade, onde o processo de decomposição ocorreu em três etapas para as amostras CaP-neutro (b) e CaP-etil (c), e em quatro etapas para a amostra CaP-base (a), com faixas de temperaturas semelhantes, para todas as amostras.

As temperaturas onset ocorreram em aproximadamente $40,375,500$ e $610^{\circ} \mathrm{C}$, para a amostra CaP-base; em 35,500 e $600^{\circ} \mathrm{C}$ para a amostra CaP-neutro e em 37,350 e $620^{\circ} \mathrm{C}$ para a amostra CaP-etil. O primeiro evento teve temperaturas máximas de decomposição em aproximadamente 47,53 e $51{ }^{\circ} \mathrm{C}$, para as amostras CaP-base, CaP-neutro e CaP-etil, respectivamente, correspondendo à perda de água adsorvida (desidratação da ligação da água da superfície de HAp). Para a amostra CaP-base a segunda e terceira etapas ocorrem em temperaturas máximas de decomposição de 443 e $553^{\circ} \mathrm{C}$, referente a perda de água da rede e à decomposição residual da matéria orgânica $[38,39]$. Para as amostras CaP-neutro e CaP-etil, a segunda etapa ocorreu com temperaturas máximas de decomposição em 545 e $531^{\circ} \mathrm{C}$, respectivamente, também referente à perda de água da rede e à decomposição residual da matéria orgânica. Em ambas as amostras, o último evento teve decomposição máxima em aproximadamente $660^{\circ} \mathrm{C}$, e está relacionado à descarbonização e início dos processos de desidroxilação [40].

As amostras tiveram perdas de massa totais praticamente iguais, sendo estas de 25,7, 25,5 e 25,6\% para as amostras CaP-base, CaP-neutro e CaP-etil, respectivamente.
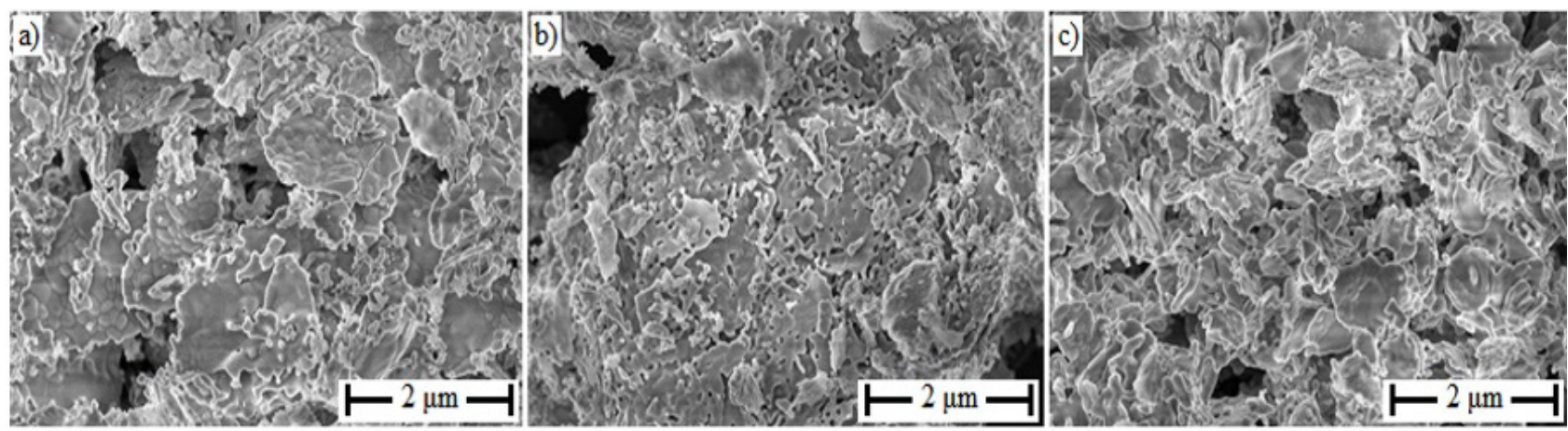

Figura 3. Imagens de MEV: (a) CaP-base; (b) CaP-neutro; e (c) CaP-etil. 


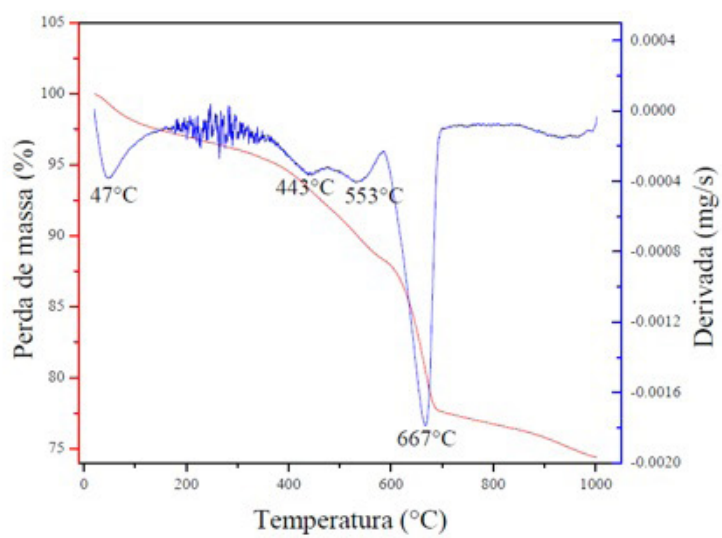

(a)

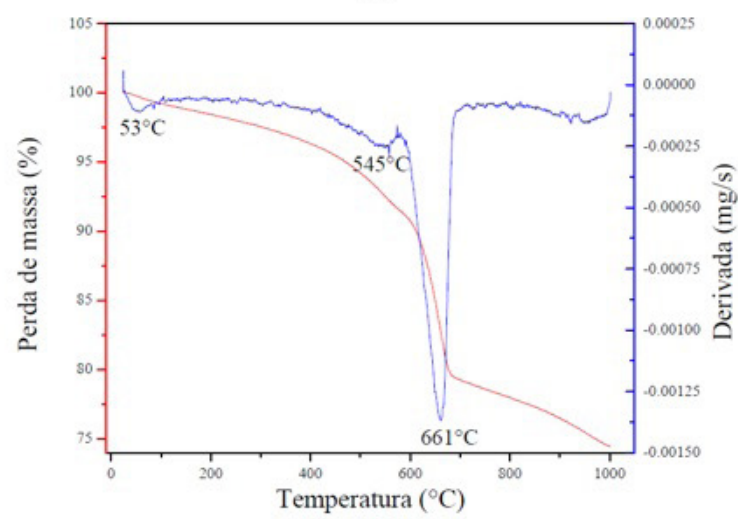

(b)

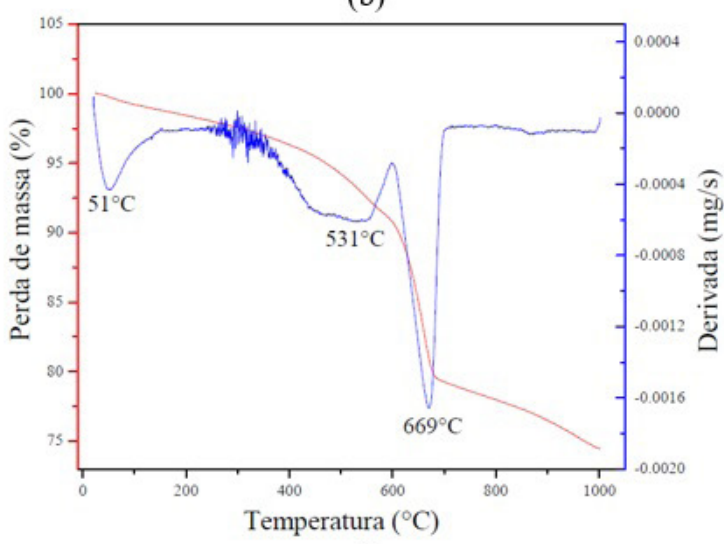

(c)

Figura 4. Análise termogravimétrica amostras CaP-base (a), CaPneutro (b) e CaP-etil (c).

\section{Conclusões}

Diante dos resultados obtidos verificou-se a obtenção de uma cerâmica bifásica de HAp e $\beta$-TCP utilizado o método Pechini, mesmo utilizando diferentes solventes na síntese (água e álcool etílico), alcançando o objetivo principal, proposto no trabalho.

Analisando o uso da água com $\mathrm{pH}$ básico como solvente na síntese, obteve-se uma amostra com uma quantificação da fase HAp maior (73\%) em relação a fase $\beta$-TCP (24\%), uma maior índice de cristalinidade (96\%) e tamanho de cristalino cerca de $53,6 \mathrm{~nm}$, se comparado com os demais solventes (água com pH neutro e álcool etílico). Porém, com a utilização do álcool etílico como solvente, observou-se que este acelera o processo de síntese favorecendo a redução da fase HAp (64\%) sendo esta a amostra que deteve menor quantificação dessa fase e a maior com $\beta$-TCP.

Todas as amostras produzidas, foram constituídas de partículas altamente reativas, formando agregados com certa porosidade, e com perdas de massa total de aproximadamente $25 \%$, quando analisadas sob temperaturas e taxas controladas.

De acordo com estudos realizados a biocerâmica de fosfato de cálcio bifásico (HAp/ $\beta$-TCP) é promissora, revolucionando a tecnologia biomédica. Tudo isso devido as propriedades da associação entre HAp/ $\beta$-TCP se mostrarem superiores, se comparado com amostras que possuem apenas fases isoladas de HAp ou $\beta$-TCP. Assim esse artigo teve como motivação inicial obter BCP por meio do método Pechini, sendo este pouco estudado, até o momento, para sinterização de tais biocerâmicas.

\section{Agradecimentos}

Os autores agradecem pelo apoio e realização dos experimentos ao Laboratório Interdisciplinar de Materiais Avançados da Universidade Federal do Piauí (LIMAV/UFPI), ao Laboratório de Tecnologia de Materiais da Universidade Federal de Campina Grande (LTM/UFCG) e ao Laboratório de Fisica dos Materiais da Universidade Federal do Piauí (FISMAT/UFPI).

\section{Referências}

1 Cheng L, Shi Y, Ye F, Bu H. Osteoinduction of calcium phosphate biomaterials in small animals. Materials Science and Engineering C. 2013;33(3):1254-1260.

2 Cheung HS, Haak MH. Growth of osteoblasts on porous calcium phosphate ceramic: an in vitro model for biocompatibility study. Biomaterials. 1989;10(1):63-67.

3 Zhu Y, Zhang K, Zhao R, Ye X, Chen X, Xiao Z, et al. Bone regeneration with micro/nano hybrid-structured biphasic calcium phosphate bioceramics at segmental bone defect and the induced immunoregulation of MSCs. Biomaterials. 2017;147:133-144. 
4 Gómez-Barrena E, Rosset P, Gebhard F, Hernigou P, Baldini N, Rouard H, et al. Feasibility and safety of treating non-unions in tibia, femur and humerus with autologous, expanded, bone marrow-derived mesenchymal stromal cells associated with biphasic calcium phosphate biomaterials in a multicentric, non-comparative trial. Biomaterials. 2019;196:100-108.

5 Bouler JM, Pilet P, Gauthier O, Verron E. Biphasic calcium phosphate ceramics for bone reconstruction: A review of biological response. Acta Biomaterialia. 2017;53:1-12.

6 Shim KS, Kim HJ, Kim SE, Park K. Park k. Simple surface biofunctionalization of biphasic calcium phosphates for improving osteogenic activity and bone tissue regeneration. Journal of Industrial and Engineering Chemistry. 2018;68:220-228.

7 Neto AS, Fonseca AC, Abrantes JCC, Coelho JFJ, Ferreira JMF. Surface functionalization of cuttlefish bonederived biphasic calcium phosphate scaffolds with polymeric coatings. Materials Science and Engineering C. 2019;105:110014.

8 Ebrahimi M, Botelho MG, Dorozhkin SV. Biphasic calcium phosphates bioceramics (HA/TCP): Concept, physicochemical properties and the impact of standardization of study protocols in biomaterials research. Materials Science and Engineering C. 2017;71:1293-1312.

9 Jmal N, Bouaziz J. Synthesis, characterization and bioactivity of a calcium-phosphate glass-ceramics obtained by the sol-gel processing method. Materials Science and Engineering C. 2017;71:279-288.

10 Cheng K, Zhang S, Weng WJ. Sol-gel prepared $\beta$-TCP/FHA biphasic coatings. Thin Solid Films. 2006;515(1):135140.

11 Chen JD, Wang YJ, Chen XF, Ren L, Lai C, He W, et al. A simple sol-gel technique for synthesis of nanostructured hydroxyapatite, tricalcium phosphate and biphasic powders. Materials Letters. 2011;65(12):1923-1926.

12 Onoda H, Yamazaki S. Homogenous hydrothermal synthesis of calcium phosphate with calcium carbonate and corbicula shells. Journal of Asian Ceramic Societies. 2016;4(4):403-406.

13 Higuita LP, Vargas AF. Effect of addition of calcium ions and hydrothermal treatment on the morphology of calcium phosphates. Materials Letters. 2017;190:146-149.

14 Titorenkova R, Dyulgerova E, Petkova V, Ilieva R. Carbonation and dehydroxylation of apatite during high energy milling of biphasic Ca-phosphate ceramics. Ceramics International. 2019;45(6):7025-7033.

15 Mohammadi Z, Mesgar ASM, Disfani FR. Preparation and characterization of single phase, biphasic and triphasic calcium phosphate whisker-like fibers by homogeneous precipitation using urea. Ceramics International. 2016;42(6):6955-6961.

16 Webler GD, Zapata MJM, Agra LC, Barreto E, Silva AOS, Hickmann JM, et al. Characterization and evaluation of cytotoxicity of biphasic calcium phosphate synthesized by a solid state reaction route. Current Applied Physics. 2014;14(6):876-880.

17 Ho WF, Hsu HC, Hsu SK, Hung CW, Wu SC. Calcium phosphate bioceramics synthesized from eggsshell powders through a solid state reaction. Ceramics International. 2013;39(6):6467-6473.

18 Karimi M, Hesaraki S, Alizadeh M, Kazemzadeh A. Synthesis of calcium phosphate nanoparticles in deep-eutectic choline chloride-urea medium: Investigating the role of synthesis temperature on phase characteristics and physical properties. Ceramics International. 2016;42(2):2780-2788.

19 Drevet R, Jaber NB, Fauré J, Tara A, Larbi BC, Benhayoune H. Electrophoretic deposition (EPD) of nanohydroxyapatite coatings with improved mechanical properties on prosthetic Ti6Al4V substrates. Surface and Coatings Technology. 2016;301:94-99.

20 Omori Y, Okada M, Takeda S, Matsumoto N. Fabrication of dispersible calcium phosphate nanocrystals via a modified Pechini method under non-stoichiometric conditions. Materials Science and Engineering C. 2014;42:562568.

21 Guo R, Huang J, Chen X, Luo Q, Luo L, Xiong Y, et al. Pechini sol-gel synthesis of La2CaB8O16:Eu3+ red phosphor and its photoluminescence spectral properties. Journal of Luminescence. 2019;206:15-20.

22 Scherrer P. Bestimmung der grosse und der inneren struktur von kolloidteilchen mittels rontgenstrahlen. Nachrichten von der Königlichen Gesellschaft der Wissenschaften zu Göttingen. 1918;3:98-100.

23 Pechini MP. Method of preparing lead and alkaline earth titanates and niobates and coating method using the same to form a capacitor. United States patent US 3330697. 1967. 
24 Aminzare M, Eskandari A, Baroonian MH, Berenov A, Razavi Hesabi Z, Taheri M, et al. Hydroxyapatite nanocomposites: synthesis, sintering and mechanical properties. Ceramics International. 2013;39(3):2197-2206.

25 Santos ML, Florentino AO, Saeki MJ, Aparecida AH, Fook MVL, Guastaldi AC. Síntese de hidroxiapatita pelo método sol gel utilizando precursores alternativos: nitrato de cálcio e ácido fosfórico. Eclética Química. 2005;3(30):29-35.

26 Roopalakshmi S, Ravishankar R, Belaldavar S, Prasad RGSV, Phani AR. Investigation of structural and morphological characteristics of hydroxyapatite synthesized by sol-gel process. Materials Today: Proceedings. 2017;4:12026-12031.

27 Chakraborty R, Seesala VS, Sen M, Sengupta S, Dhara S, Saha P, et al. MWCNT reinforced bone like calcium phosphate: hydroxyapatite composite coating developed through pulsed electrodeposition with varying amount of apatite phase and crystallinity to promote superior osteoconduction, cytocompatibility and corrosion protection performance compared to bare metallic implant surface. Surface and Coatings Technology. 2017;325(25):496-514.

28 Wang J, Su Y, Xu L, Li D. Fabrication and preliminary evaluation of the osteogenic potential for micro-/nanostructured porous BCP ceramics. Ceramics International. 2020;46(4):4801-4812.

29 Raynaud S, Champion E, Bernache-Assollant D, Thomas P. Calcium phosphate apatites with variable Ca/P atomic ratio I. Synthesis, characterisation and thermal stability of powders. Biomaterials. 2002;23(4):1065-1072.

30 Stötzel C, Müller FA, Reinert F, Niederdraenk F, Barralet JE, Gbureck U. Ion adsorption behaviour of hydroxyapatite with different crystallinities. Colloids and Surfaces. B, Biointerfaces. 2009;74(1):91-95.

31 Dorozhkin SV. Calcium orthophosphates as bioceramics: state of the art. Journal of Functional Biomaterials. 2010;31(7):1465-1485.

32 Phatai P, Futalan CM, Utara S, Khemthong P, Kamonwannasit S. Structural characterization of cerium-doped hydroxyapatite nanoparticles synthesized by ultrasonic-assisted sol-gel technique. Results in Physics. 2018;10:956963.

33 Rameshbabu N, Kumar TSS, Prabhakar TG, Sastry VS, Murty KVGK, Rao KP. Antibacterial nanosized silver substituted hydroxyapatite: synthesis and characterization. Journal of Biomedical Materials Research. Part A. 2007;80(3):581-591.

34 Ben-Arfa BAE, Fernandes HR, Miranda Salvado IM, Ferreira JMF, Pullar RC. Synthesis andbioactivity assessment of high silica content quaternary glasses with $\mathrm{Ca}$ : P ratios of 1.5 and 1.67, made by a rapid sol-gelprocess. Journal of Biomedical Materials Research. Part A. 2017;70:796-804.

35 Piazza RD, Pelizaro TAG, Rodriguez-Chanfrau JE, Serna AAL, Veranes-Pantoja Y, Guastaldi AC. Calcium phosphates nanoparticles: the effect of freeze-drying on particle size reduction. Materials Chemistry and Physics. 2020;239:122004.

36 Pei L, Zhang X, Zhang L, Zhang Y, Xu Y. Solvent influence on the morphology and supercapacitor performance of the nickel oxide. Materials Letters. 2016;162:238-241.

37 Sofronia AM, Baies R, Anghel EM, Marinescu CA, Tanasescu S. Thermal and structural characterization of synthetic and natural nanocrystalline hydroxyapatite. Materials Science and Engineering. 2014;43:153-163.

38 Manjubala I, Sivakumar M. In-situ synthesis of biphasic calcium phosphate ceramics using microwave irradiation. Materials Chemistry and Physics. 2001;71(3):272-278.

39 Londoño-Restrep SM, Zubieta-Otero LF, Jeronimo-Cruz R, Mondragon MA, Rodriguez-García ME. Effect of the crystal size of biogenic hydroxyapatites on IR and Raman spectra. Materials Science and Engineering. 2014;43:153163.

40 Sofronia AM, Baies R, Anghel EM, Marinescu CA, Tanasescu S. Thermal and structural characterization of synthetic and natural nanocrystalline hydroxyapatite. Materials Science and Engineering: C. 2014;43:153-163.

Recebido em: 26 Abr. 2020

Aceito em: 17 Out. 2020 